\title{
Symmetry reductions of a $2+1$ Lax pair
}

\section{P. G. Estévez.}

\section{Dept. Fisica Fundamental. University of Salamanca SPAIN}

in collaboration with J. Prada and M. L. Gandarias

Salamanca 2007 
The identification of the Lie symmetries of a PDE is an instrument of primary importance in order to solve such an equation. A standard method for finding solutions of PDEs is that of reduction using Lie symmetries:

Each Lie symmetry allows a reduction of the PDE to a new equation with the number of independent variables reduced by one

this procedure gives rise to the ARS conjecture which establishes that a PDE is integrable in the Painlevé sense if all its reductions pass the Painlevé test.

This means that solutions of a PDE can be achieved by solving its reductions to ordinary differential equations (ODE).

Classical and non-classical (Olver, Bluman, Cole) Lie symmetries are the usual way for identifying the reductions. 
Nevertheless, let us recall that there are some methods for solving PDEs that are more effective in $2+1$ than in $1+1$

\section{dimensions.}

- The Singular Manifold Method (Weiss) based on the Painlevé property requires the truncation of the Painlevé series at the constant level. Sometimes this condition is very restrictive and must be modified (Estevez-Leble 1995).

- For some equations in $1+1$ dimensions the SMM imposes so many restrictions that there is not freedom enough to get nontrivial solutions and/or to introduce a spectral parameter (Estevez et al 1993).

- Nevertheless, these problems disappear when we pass to higher dimensions. It is necessary to increase the number of dimensions in order to have sufficient freedom so that the SMM will not be too restrictive. 
A good example of the above statement is the following equation in $2+1$ dimensions

$$
\left[h_{x x z}-\frac{3}{4}\left(\frac{h_{x z}^{2}}{h_{z}}\right)+3 h_{x} h_{z}\right]_{x}=h_{y z}
$$

which we have studied in a recent paper (JNMP 2005) proving that the SMM is a very effective method for solving the equation. It is quite straightforward to determine the associated linear problem through the SMM.

$$
\begin{aligned}
& -\psi_{y}+\psi_{x x x}+3 h_{x} \psi_{x}+\frac{3}{2} h_{x x} \psi=0, \\
& 2 h_{z} \psi_{x z}-h_{x z} \psi_{z}+2 h_{z}^{2} \psi=0,
\end{aligned}
$$


The SMM also provides Darboux transformations and an iterative method to construct solutions. Nevertheless, different reductions of our equation) generate a lot of problems when we attempt to apply the SMM to them (JNMP 2005).

\section{This means that the SMM allows us to solve the PDE in} $2+1$ dimensions but not their reductions to $1+1$.

Therefore, our plan in this paper is, to a certain extent, exactly the opposite of the usual approach: we try to obtain $1+1$ spectral problems arising from a $2+1$ Lax pair. 
- Once we have solved the problem in $2+1$ dimensions, in the sense that we have determined its Lax pair, we shall identify the classical symmetries of the Lax pair.

- We use these symmetries to obtain a reduced Lax pair in $1+1$ dimensions whose compatibility condition should be a reduction of the equation. The $2+1$ Lax pair does not contain a spectral parameter but the reduction process should introduce a spectral parameter that is absolutely essential in $1+1$ dimensions.

- Actually, there are five possible reductions. Three of them yield linear equations that can be easily integrated. The other two reductions yield $1+1$ spectral problems that include, well known equations.

- It is interesting to note that each of these reductions yields respectively two, three and fourth order spectral problems. 


\section{Classical Symmetries}

In order to apply the Classical Lie Method to the system of PDEs with three independent variables and two fields, we consider the oneparameter following Lie group of infinitesimal transformations in $x, y, z, h, \psi$ :

$$
\begin{aligned}
x^{\prime} & =x+\varepsilon \xi_{1}(x, y, z, h, \psi)+O\left(\varepsilon^{2}\right) \\
y^{\prime} & =y+\varepsilon \xi_{2}(x, y, z, h, \psi)+O\left(\varepsilon^{2}\right) \\
z^{\prime} & =z+\varepsilon \xi_{3}(x, y, z, h, \psi)+O\left(\varepsilon^{2}\right) \\
h^{\prime} & =h+\varepsilon \phi_{1}(x, y, z, h, \psi)+O\left(\varepsilon^{2}\right) \\
\psi^{\prime} & =\psi+\varepsilon \phi_{2}(x, y, z, h, \psi)+O\left(\varepsilon^{2}\right)
\end{aligned}
$$

where $\epsilon$ is the group parameter. It is therefore necessary that this one transformation leaves the set of solutions invariant. This yields an overdetermined linear system of equations for the infinitesimals $\xi_{1}, \xi_{2}$, $\xi_{3}, \phi_{1}$ and $\phi_{2}$. 
The associated Lie algebra of infinitesimal symmetries is the set of vector fields of the form:

$$
X=\xi_{1} \frac{\partial}{\partial x}+\xi_{2} \frac{\partial}{\partial y}+\xi_{3} \frac{\partial}{\partial z}+\phi_{1} \frac{\partial}{\partial h}+\phi_{2} \frac{\partial}{\partial \psi}
$$

By applying the classical method, we obtain the following generators:

$$
\begin{aligned}
\xi_{1} & =-2 \frac{d A_{1}(y)}{d y} x-\frac{3}{2} A_{2}(y) \\
\xi_{2} & =-6 A_{1}(y) \\
\xi_{3} & =\beta(z) \\
\phi_{1} & =\frac{1}{3} \frac{d^{2} A_{1}(y)}{d y^{2}} x^{2}+\frac{1}{2} \frac{d A_{2}(y)}{d y} x+A_{3}(y)+2 \frac{d A_{1}(y)}{d y} h \\
\phi_{2} & =\left(\lambda+\frac{d A_{1}(y)}{d y}\right) \psi
\end{aligned}
$$

The corresponding Lie symmetry algebra depends on three $A_{1}(y)$, $A_{2}(y)$ and $A_{3}(y)$ arbitrary functions of $y$ and an arbitrary function $\beta(z)$ of $z$. The only constant that appears is $\lambda$ that, as we shall show, plays the role of the spectral parameter in the $1+1$ reductions. 
Having determined the infinitesimals, the symmetry variables are found by solving the invariant surface conditions

$$
\begin{aligned}
& \Phi_{1} \equiv \xi_{1} \frac{\partial h}{\partial x}+\xi_{2} \frac{\partial h}{\partial y}+\xi_{3} \frac{\partial h}{\partial z}-\phi_{1}=0 \\
& \Phi_{2} \equiv \xi_{1} \frac{\partial \psi}{\partial x}+\xi_{2} \frac{\partial \psi}{\partial y}+\xi_{3} \frac{\partial \psi}{\partial z}-\phi_{2}=0
\end{aligned}
$$

or the corresponding characteristic equations

$$
\begin{aligned}
& \frac{-d x}{2 \frac{d A_{1}}{d y} x+\frac{3}{2} A_{2}}=\frac{-d y}{6 A_{1}}=\frac{d z}{\beta}= \\
= & \frac{d h}{\frac{1}{3} \frac{d^{2} A_{1}}{d y^{2}} x^{2}+\frac{1}{2} \frac{d A_{2}}{d y} x+A_{3}+2 \frac{d A_{1}}{d y} h}=\frac{d \psi}{\left(\lambda+\frac{d A_{1}}{d y}\right) \psi}
\end{aligned}
$$

There are different possibilities. 


\section{Reductions}

There are five independent reductions that we determine in the following way 


\section{Case 1) $A_{1} \neq 0, \quad \beta \neq 0$}

The reduced variables are:

$$
z_{1}=\frac{x}{A_{1}^{1 / 3}}-\frac{1}{4} \int \frac{A_{2}}{A_{1}^{4 / 3}} d y, \quad z_{2}=6 \int \frac{1}{\beta} d z+\int \frac{1}{A_{1}} d y
$$

and the following reduction for the fields

$$
\begin{aligned}
\psi(x, y, z) & =\frac{e^{-\frac{\lambda}{6} \int \frac{d y}{A_{1}}}}{A_{1}^{1 / 6}} G\left(z_{1}, z_{2}\right) \\
h(x, y, z) & =\frac{1}{A_{1}^{1 / 3}} U\left(z_{1}, z_{2}\right)-\frac{1}{6 A_{1}^{1 / 3}} \int A_{1}^{1 / 3} M\left(z_{1}, y\right) d y
\end{aligned}
$$


where $M\left(z_{1}, y\right)$ is the function

$$
\begin{aligned}
& M\left(z_{1}, y\right)=\left(\frac{d^{2} A_{1}}{d y^{2}} \frac{1}{3 A_{1}^{1 / 3}}\right) z_{1}^{2}+ \\
+ & \left(\frac{d A_{2}}{d y} \frac{1}{2 A_{1}^{2 / 3}}+\frac{d^{2} A_{1}}{d y^{2}} \frac{1}{6 A_{1}^{1 / 3}} \int \frac{A_{2}}{A_{1}^{4 / 3}} d y\right) z_{1}+ \\
+ & \frac{d^{2} A_{1}}{d y^{2}} \frac{1}{48 A_{1}^{1 / 3}}\left(\int \frac{A_{2}}{A_{1}^{4 / 3}} d y\right)^{2}+\frac{d A_{2}}{d y} \frac{1}{8 A_{1}^{2 / 3}} \int \frac{A_{2}}{A_{1}^{4 / 3}} d y+\frac{A_{3}}{A_{1}}
\end{aligned}
$$

Substitution of the reduction ansatz gives us:

$$
\begin{aligned}
& 0=G_{z_{1} z_{1} z_{1}}-G_{z_{2}}+3 U_{z_{1}} G_{z_{1}}+\frac{3}{2} U_{z_{1} z_{1}} G+\frac{\lambda}{6} G \\
& 0=2 U_{z_{2}} G_{z_{2} z_{1}}-U_{z_{2} z_{1}} G_{z_{2}}+2 U_{z_{2}}^{2} G
\end{aligned}
$$


Solving for $G_{z_{2}}$, we obtain the fourth order spectral problem:

$$
\begin{aligned}
G_{z_{2}} & =G_{z_{1} z_{1} z_{1}}+3 U_{z_{1}} G_{z_{1}}+\frac{3}{2} U_{z_{1} z_{1}} G+\frac{\lambda}{6} G \\
U_{z_{2}} G_{z_{1} z_{1} z_{1} z_{1}} & =\frac{1}{2} U_{z_{2} z_{1}} G_{z_{1} z_{1} z_{1}}-3 U_{z_{1}} U_{z_{2}} G_{z_{1} z_{1}}+ \\
& +\left(\frac{3}{2} U_{z_{1}} U_{z_{2} z_{1}}-\frac{\lambda}{6} U_{z_{2}}-\frac{9}{2} U_{z_{1} z_{1}} U_{z_{2}}\right) G_{z_{1}}+ \\
& +\left(\frac{\lambda}{12} U_{z_{2} z_{1}}-U_{z_{2}}^{2}-\frac{3}{2} U_{z_{1} z_{1} z_{1}} U_{z_{2}}+\frac{3}{4} U_{z_{1} z_{1}} U_{z_{2} z_{1}}\right) G
\end{aligned}
$$

whose compatibility condition gives us the $1+1$ equation

$$
\left(U_{z_{2} z_{1} z_{1}}-\frac{3}{4} \frac{U_{z_{2} z_{1}}^{2}}{U_{z_{2}}}+3 U_{z_{2}} U_{z_{1}}\right)_{z_{1}}=U_{z_{2} z_{2}}
$$

that can be written as the Drinfel'd Sokolov nonlocal $\mathrm{KdV}$ equation 


$$
z_{1}=\frac{x}{A_{1}^{1 / 3}}-\frac{1}{4} \int \frac{A_{2}}{A_{1}^{4 / 3}} d y, \quad z_{2}=z
$$

and the reduction for the fields is exactly the same as in case 1 . We obtain the third order spectral problem:

$$
\begin{aligned}
0 & =G_{z_{1} z_{1} z_{1}}+3 U_{z_{1}} G_{z_{1}}+\frac{3}{2} U_{z_{1} z_{1}} G+\frac{\lambda}{6} G \\
0 & =2 U_{z_{2}} G_{z_{2} z_{1}}-U_{z_{2} z_{1}} G_{z_{2}}+2 U_{z_{2}}^{2} G
\end{aligned}
$$

whose compatibility gives us the $1+1$ equation

$$
\left(U_{z_{2} z_{1} z_{1}}-\frac{3}{4} \frac{U_{z_{2} z_{1}}^{2}}{U_{z_{2}}}+3 U_{z_{2}} U_{z_{1}}\right)_{z_{1}}=0
$$


An alternative form of the above equation arises from the following definitions

$$
U_{z_{1}}=\frac{4}{3} V, \quad U_{z_{2}}=-P^{2}
$$

which allow us to write it as the system

$$
P P_{z_{1} z_{1}}-\frac{P_{z_{1}}^{2}}{2}+2 P^{2} V+F\left(z_{2}\right)=0
$$

$$
V_{z_{2}}=-\frac{3}{4}\left(P^{2}\right)_{z_{1}}
$$

where $F\left(z_{2}\right)$ is an arbitrary function. It is the Ermakov-Pinney equation that is related through a reciprocal transformation to the DegasperisProcesi equation. 
Case 3) $A_{1}=0, \quad A_{2} \neq 0, \quad \beta \neq 0$

$$
\begin{aligned}
z_{1} & =x+\frac{3}{2} A_{2} \int \frac{1}{\beta} d z, \quad z_{2}=y \\
\psi(x, y, z) & =e^{\left(-\frac{2 \lambda}{3 A_{2}}\right) x} G\left(z_{1}, z_{2}\right) \\
h(x, y, z) & =U\left(z_{1}, z_{2}\right)-\frac{1}{6} \frac{\frac{d^{2} A_{2}}{d y^{2}}}{A_{2}} x^{2}-\frac{2 A_{3}}{3 A_{2}} x
\end{aligned}
$$

Substitution of the reduction ansatz gives us:

$$
\begin{aligned}
0 & =G_{z_{1} z_{1} z_{1}}-G_{z_{2}}-\frac{2 \lambda}{A_{2}} G_{z_{1} z_{1}}+\left(3 U_{z_{1}}+\frac{4 \lambda^{2}}{3 A_{2}^{2}}-\frac{2 A_{3}}{A_{2}}-\frac{d A_{2}}{d z_{2}} \frac{z_{1}}{A_{2}}\right) G_{z_{1}} \\
& +\left(\frac{3}{2} U_{z_{1} z_{1}}-\frac{2 \lambda}{A_{2}} U_{z_{1}}+\frac{4 \lambda A_{3}}{3 A_{2}^{2}}-\frac{8 \lambda^{3}}{27 A_{2}^{3}}-\frac{1}{2 A_{2}} \frac{d A_{2}}{d z_{2}}\right) G \\
0 & =U_{z_{1}} G_{z_{1} z_{1}}-\left(\frac{1}{2} U_{z_{1} z_{1}}+\frac{2 \lambda}{3 A_{2}} U_{z_{1}}\right) G_{z_{1}}+U_{z_{1}}^{2} G
\end{aligned}
$$


Solving for $G_{z_{1} z_{1}}$, we obtain the second order linear system:

$$
\begin{aligned}
G_{z_{1} z_{1}} & =\left(\frac{2 \lambda}{3 A_{2}}+\frac{U_{z_{1} z_{1}}}{2 U_{z_{1}}}\right) G_{z_{1}}-U_{z_{1}} G \\
G_{z_{2}} & =\left(\frac{4 \lambda^{2}}{9 A_{2}^{2}}-\frac{U_{z_{1} z_{1}}^{2}}{4 U_{z_{1}}^{2}}-\frac{\lambda}{3 A_{2}} \frac{U_{z_{1} z_{1}}}{U_{z_{1}}}-\frac{z_{1}}{A_{2}} \frac{d A_{2}}{d z_{2}}\right) G_{z_{1}}+ \\
& +\left(\frac{U_{z_{1} z_{1} z_{1}}}{2 U_{z_{1}}}+2 U_{z_{1}}-2 \frac{A_{3}}{A_{2}^{2}}\right) G_{z_{1}}+ \\
& +\left(\frac{4 \lambda A_{3}}{3 A_{2}^{2}}-\frac{2 \lambda}{3 A_{2}} U_{z_{1}}-\frac{8 \lambda^{3}}{27 A_{2}^{3}}-\frac{1}{2 A_{2}} \frac{d A_{2}}{d z_{2}}\right) G
\end{aligned}
$$


whose compatibility condition yields to the $1+1$ equation

$$
\left(U_{z_{2}}-U_{z_{1} z_{1} z_{1}}+\frac{3}{4} \frac{U_{z_{1} z_{1}}^{2}}{U_{z_{1}}}-3 U_{z_{1}}^{2}+\frac{2 A_{3}}{A_{2}} U_{z_{1}}+\frac{\left(z_{1} U_{z_{1}}+U\right)}{A_{2}} \frac{d A_{2}}{d z_{2}}\right)_{z_{1}}=0
$$

Setting

$$
U_{z_{1}}=-\left(\frac{Q(T, X)}{A_{2}}\right)^{2}, \quad T=\int \frac{1}{A_{2}^{3}} d z_{2}, \quad X=\frac{z_{1}}{A_{2}}-2 \int \frac{A_{3}}{A_{2}^{2}} d z_{2}
$$

we have the Modified Korteveg-de Vries equation

$$
Q_{T}-Q_{X X X}+6 Q^{2} Q_{X}=0
$$




$$
\begin{aligned}
z_{1} & =y, \quad z_{2}=z \\
\psi(x, y, z) & =e^{-\frac{2 \lambda}{3 A_{2}} x} G\left(z_{1}, z_{2}\right) \\
h(x, y, z) & =U\left(z_{1}, z_{2}\right)-\frac{1}{6 A_{2}} \frac{d A_{2}}{d y} x^{2}-\frac{2}{3} \frac{A_{3}}{A_{2}} x
\end{aligned}
$$

whose substitution gives us

$$
G_{z_{1}}=\left(\frac{4 \lambda A_{3}}{3 A_{2}^{2}}-\frac{8 \lambda^{3}}{27 A_{2}^{3}}-\frac{1}{A_{2}} \frac{d A_{2}}{d z_{1}}\right) G, \quad G_{z_{2}}=\frac{3 A_{2}}{2 \lambda} U_{z_{2}} G
$$

whose compatibility provides the linear equation

$$
A_{2} U_{z_{1} z_{2}}+\frac{d A_{2}}{d z_{1}} U_{z_{2}}=0 \Longrightarrow U\left(z_{1}, z_{2}\right)=\frac{K_{1}\left(z_{2}\right)}{A_{2}}+F_{1}\left(z_{1}\right)
$$

where $F_{1}\left(z_{1}\right)$ and $K_{1}\left(z_{2}\right)$ are arbitrary functions. We can integrate as:

$$
G=\alpha_{0} \frac{1}{\sqrt{A_{2}}} e^{\frac{3 K_{1}}{2 \lambda}+\frac{4 \lambda}{3} \int \frac{A_{3}}{A_{2}^{2}} d z_{1}-\frac{8 \lambda^{3}}{27} \int \frac{1}{A_{2}^{3}} d z_{1}}
$$


where $\alpha_{0}$ is an arbitrary constant. The resulting solution is

$$
\begin{aligned}
& h=\frac{K_{1}}{A_{2}}+F_{1}-\frac{1}{6 A_{2}} \frac{d A_{2}}{d y} x^{2}-\frac{2}{3} \frac{A_{3}}{A_{2}} x \\
& \psi=\frac{\alpha_{0}}{\sqrt{A_{2}}} e^{\frac{3 K_{1}}{2 \lambda}+\frac{4 \lambda}{3} \int \frac{A_{3}}{A_{2}^{2}} d y-\frac{8 \lambda^{3}}{27} \int \frac{1}{A_{2}^{3}} d y-\frac{2 \lambda}{3 A_{2}} x}
\end{aligned}
$$

depending on two arbitrary constants $\alpha_{0}$ and $\lambda$ and five arbitrary functions $A_{1}(y), A_{2}(y), A_{3}(y), F_{1}(y)$, and $K_{1}(z)$. 


$$
\begin{aligned}
z_{1} & =x, \quad z_{2}=y \\
\psi(x, y, z) & =e^{\lambda \int \frac{d z}{\beta}} G\left(z_{1}, z_{2}\right), \quad h(x, y, z)=U\left(z_{1}, z_{2}\right)+A_{3} \int \frac{d z}{\beta}
\end{aligned}
$$

Substitution of the reduction ansatz gives us:

$$
0=G_{z_{1} z_{1} z_{1}}-G_{z_{2}}+3 U_{z_{1}} G_{z_{1}}+\frac{3}{2} U_{z_{1} z_{1}} G, \quad 0=\lambda G_{z_{1}}+A_{3} G
$$

The compatibility condition yields to the linear equation

$$
\lambda U_{z_{1} z_{1} z_{1}}-2 A_{3} U_{z_{1} z_{1}}+\frac{2}{3} \frac{d A_{3}}{d z_{2}}=0
$$

that can be easily integrated as:

$$
\begin{aligned}
U\left(z_{1}, z_{2}\right) & =F_{3}\left(z_{2}\right) e^{\frac{2 A_{3}}{\lambda} z_{1}}+\frac{1}{6 A_{3}} \frac{d A_{3}}{d z_{2}} z_{1}^{2}+\left(\frac{\lambda}{6 A_{3}^{2}} \frac{d A_{3}}{d z_{2}}+\frac{1}{2 A_{3}} F_{1}\left(z_{2}\right)\right) z_{1} \\
& +\frac{\lambda^{2}}{12 A_{3}^{3}} \frac{d A_{3}}{d z_{2}}+\frac{\lambda}{4 A_{3}^{2}} F_{1}\left(z_{2}\right)+\frac{1}{2 A_{3}} F_{2}\left(z_{2}\right)
\end{aligned}
$$

where $F_{1}\left(z_{2}\right), F_{2}\left(z_{2}\right)$ and $F_{3}\left(z_{2}\right)$ are arbitrary functions. 


$$
G=\alpha_{0} e^{-\left(\frac{3}{2 \lambda} \int F_{1} d z_{2}+\frac{1}{\lambda^{3}} \int A_{3}^{3} d z_{2}+\frac{A_{3}}{\lambda} z_{1}\right)}
$$

where $\alpha_{0}$ is an arbitrary constant. We obtain the following solution:

$$
\begin{aligned}
& h=F_{3} e^{\frac{2 A_{3}}{\lambda} x}+\frac{1}{6 A_{3}} \frac{d A_{3}}{d y} x^{2}+\left(\frac{\lambda}{6 A_{3}^{2}} \frac{d A_{3}}{d y}+\frac{1}{2 A_{3}} F_{1}\right) x \\
& +\frac{\lambda^{2}}{12 A_{3}^{3}} \frac{d A_{3}}{d y}+\frac{\lambda}{4 A_{3}^{2}} F_{1}+\frac{1}{2 A_{3}} F_{2}+A_{3} \int \frac{d z}{\beta} \\
& \psi=\alpha_{0} e^{\lambda \int \frac{d z}{\beta}-\left(\frac{3}{2 \lambda} \int F_{1} d y+\frac{1}{\lambda^{3}} \int A_{3}^{3} d y+\frac{A_{3}}{\lambda} x\right)}
\end{aligned}
$$

depending on two arbitrary constants $\alpha_{0}$ and $\lambda$ and six arbitrary functions $A_{1}(y), A_{2}(y), A_{3}(y), F_{1}(y), F_{2}(y), F_{3}(y)$ and $\beta(z)$. 


\section{Conclusions}

- A spectral problem in $2+1$ dimensions is presented. It should be noted that this Lax pair was obtained using the SMM that, surprisingly, does not work properly for some $1+1$ reductions of the system. It is easier to solve the problem in $2+1$ than in $1+1$ dimensions

- For the above reason we attempt to go from $2+1$ to $1+1 \mathrm{di}$ mensions by using the reductions arising from the Classical Lie symmetries of the Lax pair. This means that we obtain symmetries that are symmetries of both the field $h$ and the eigenfunction $\psi$. The symmetries that we have obtained include several arbitrary functions as well as an arbitrary constant that plays the role of the spectral parameter of the reduced spectral problems. 
- Five possible reductions arise from the Classical Symmetries. Three of them yield linear equations that can be easily integrated, providing us with nontrivial solutions.

- The other three reductions yield interesting Lax pairs of second, third and fourth order respectively. The introduction of the spectral parameters in the $1+1$ reductions arise in an absolutely natural way. 
- Ablowitz M J, Ramani A and Segur H, Lett. Nuov. Cim. 23, 1978, 333-338.

- Bluman G. W. and Cole J D, Similarity Methods for Differential Equations, Springer Verlag, 1974.

- Degasperis A and Procesi M, Asymptotic integrability. Symmetry and Perturbation Theory, G Gaeta, World Scientific, 1999, 23-37.

- Estévez P G, Gordoa P R, Martinez-Alonso L and Medina-Reus E, J. Phys A: Math. and Gen. 26, 1993, 1915-1925.

- Estévez P G and Leble S, Inv. Prob. 11, 1995, 925-937. 
- P G Estévez, Phys. Lett. A171 (1992), 259-261.

- P G Estévez, Studies in Appl. Math. 95 (1995), 73-113.

- P G Estévez and J Prada, Journal of Nonlinear Math. Phys. 12 (2005), 266-279.

- Hone A N W, Phys. Lett. A263, 1999, 347-354.

- Hone A N W and Wang J P, Inverse Problems 19, 2003, 129-145.

- Legare M, Journal of Nonlinear Math. Phys. 3, 1996, 266-285.

- Olver P J, Applications of Lie Groups to Differential Equations, Springer Verlag, 1999. 
- Painlevé P., Acta mathematica, Paris, (1900).

- Stephani H, Differential equations. Their solutions using symmetries, edited by M. Mac Callum, Cambridge University Press, 1989.

- Weiss J, J. Math. Phys. 24, 1983, 1405-1413. 